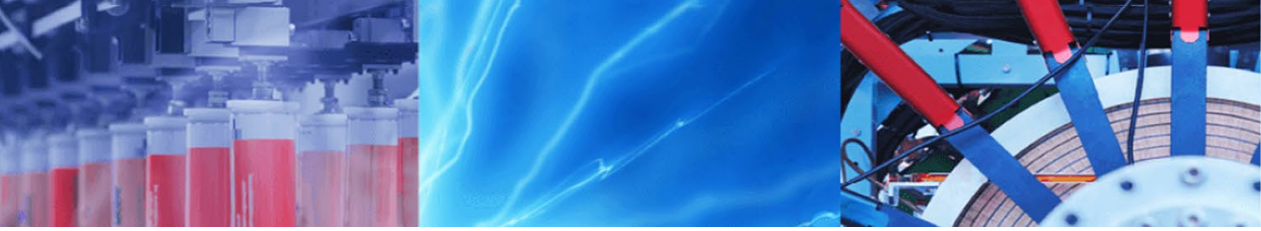

Research Article

\title{
Graph theoretical approach for screening autism on brain complex networks
}

\author{
Sima Ataei ${ }^{1}$ (D) Niousha Attar ${ }^{1} \cdot$ Sadegh Aliakbary ${ }^{1} \cdot$ Fatemeh Bakouie $^{2}$
}

() Springer Nature Switzerland AG 2019

\begin{abstract}
Autism spectrum disorder has been known as a prevalent brain disorder of children in recent years. Numerous studies have indicated differences between functional brain networks of disordered and typically developed brains. Nevertheless, no automatic and effective methodology has been established for the identification of this disorder using functional magnetic resonance images (fMRI). In this research, we investigate differences between Autistic and typically developed brain networks by modeling $\mathrm{fMRI}$ data to brain complex networks and propose a method for classification of the aforementioned groups. In this method, applying graphlet counts, as the frequency of predefined non-isomorphic subgraphs, feature vectors have been extracted and using an ensemble classifier, data has been classified into two defined groups. Results, showing $6.5 \%$ improvement according to the best baseline method, has reached $69.81 \%$ of accuracy for disorder classification.
\end{abstract}

Keywords Autism spectrum disorder · Brain networks · Complex networks · Graphlet · Classification · fMRI images

\section{Introduction}

Autism spectrum disorder (ASD) is a brain developmental disorder with signs of disabilities in social communications, recurring patterns of behavior and difficulties on speech [7]. Autism affected children cannot communicate in common manners or perceive other people's feelings and they have very limited interests. Signs of this disorder emerge before the age of 3 years old [16]. Due to ambiguous symptoms and difficulty of screening the signs on infants, needs for an automatic method for diagnosis of the disorder are increasing [6].

Human brain has been studied in various researches. One of the recent approaches is analyzing human brain as a complex network. A complex network is a collection of connected objects, modeled as a set of nodes and edges using Graph theory [10]. In a complex network perspective, a brain can be considered as a collection of brain regions, network nodes, and the connections between these regions, network edges [4]. Brain complex networks are used for investigating structural and functional abnormalcy in the human brain [11]. Any alterations in brain connections can be a potential symptom or indicator of an abnormal brain condition or disorder, investigating this alteration can lead us to diagnosis of the disorder [5].

The purpose of this research is to analyze the functional network of Autistic and Typically Developing (TD) brains and to screen the differences of these two groups on equal conditions. In this research, we are using brain network features as a criterion for detecting Autism in the brain. Since Autism disorder has a direct impact on brain functionality, using brain functional networks could be effective for screening this disorder [8]. Due to high spatial accuracy, fMRI images could be an acceptable source for investigating brain network functionality [9].

$\triangle$ Sima Ataei, si.ataei@mail.sbu.ac.ir; Niousha Attar, n_attar@sbu.ac.ir; Sadegh Aliakbary, s_aliakbary@sbu.ac.ir; Fatemeh Bakouie, f_bakouie@sbu.ac.ir| ${ }^{1}$ Faculty of Computer Science and Engineering, Shahid Beheshti University G.C., Tehran, Iran. ${ }^{2}$ Institute for Cognitive and Brain Science, Shahid Beheshti University G.C., Tehran, Iran.

SN Applied Sciences (2019) 1:1122 | https://doi.org/10.1007/s42452-019-1079-y 
In this paper, first, we review some of the related researches to this project, also a brief introduction to the dataset is provided. Then, each step of the proposed method, including functional network extraction, feature extraction, and classification are explored. Finally, in the last section, the results of the experiments are demonstrated and some discussions have been made.

\section{Related works}

Two main factors of classifying complex networks are extracted network feature vector and the type of classifier. Multiple studies have been conducted on finding the most accurate feature vector to distinguish ASD and TD brains. In [15], different network features have been introduced as a feature vector for Autistic brain network classifying. These features contain small-worldness, centrality, clustering coefficient, and modularity. Various classifiers, including K-nearest neighbor (KNN), artificial neural networks (ANN), Kernel-based methods, have been used to classify this type of data. Some other approaches try to design a suitable kernel relating to the specific problem. Dudero et al. [2] proposed a mathematical framework based on Riemannian geometry and kernel methods that could be applied to connectivity matrices for the classification tasks. They have trained and tested proposed kernel using crossvalidation method and reported the accuracy of $60.76 \%$. In [3], a new approach based on Grassmannian geometry and graph Laplacians is introduced, which permits to learn a set of sub-connectivities that can be used in combination with support vector machine (SVM) to classify functional connectomes. Applying this new method, classification accuracy improved to $63.29 \%$.

\section{Dataset}

As mentioned earlier, in this study, we used a dataset extracted from resting-state fMRI brain images to discriminate ASD and TD subjects. fMRI scans are captured in a specific time period from both groups. The subjects are asked to rest their brain but keep their consciousness. We used a publicly available dataset on USC Multimodal Connectivity Database, a web-based platform for sharing human connectome data [1]. The dataset was collected at UCLA university using a Siemens 3T Trio scanner. Scans were captured for $6 \mathrm{~min}$, with 34 slices of $4 \mathrm{~mm}$. Data preprocessing and connectivity matrix extraction are already applied to the shared dataset. UCLA dataset contains 79 connectivity matrices, including 42 ASD affected subjects and 37 TD subjects [14]. More details of the dataset are available in Table 1.
Table 1 UCLA dataset subject details [14]

\begin{tabular}{lll}
\hline Characteristic & Typically developing & Autism spectrum \\
\hline Sample size & 37 & 42 \\
Number of females & 6 & 6 \\
Age & $13.0 \pm 2.0,(9.5-17.8)$ & $13.5 \pm 2.4,(9.3-17.9)$ \\
Verbal IQ & $108.4 \pm 11.0,(86-127)$ & $103.6 \pm 12.7,(79-132)$ \\
Performance IQ & $105.2 \pm 11.9,(76-129)$ & $103.5 \pm 14.4,(72-134)$ \\
Full scale IQ & $106.8 \pm 10.0,(84-128)$ & $103.3 \pm 14.0,(79-134)$ \\
\hline
\end{tabular}

\section{Proposed method}

In this method, proper functional networks have been produced using network different densities, and a set of network features are extracted for screening Autism on these brain networks. In this research, we investigate graphlets as network features for disorder screening. Definition of graphlets will be explored in the following sections. Graphlet frequencies are calculated for each brain network and the classification feature vector is extracted from these frequencies. After normalization processes, feature vectors are presented to a classifier in order to discriminate two groups of TD and ASD data. Each of these steps will be explained separately in the following sections.

\subsection{Functional network extraction}

Four steps are to be followed in the extraction of networks from fMRI scans:

1. Defining nodes of the graph, using predefined brain regions on a specific brain Altas

2. Calculating the average BOLD signal for each brain region(or graph node)

3. Calculating and Normalizing correlation between every two nodes

4. Thresholding correlations of nodes according to the problem

For the first step, power-264 Atlas is used in defining nodes of graph [12]. Each of 264 defined regions of this Atlas is considered as a node in our brain complex networks. The average BOLD signal for voxels of each region is calculated and assumed as node BOLD signal. As a result, since $\mathrm{fMRI}$ images are in the time-series format, a sequence of BOLD signals for each node is available. In the third step, the correlation between every two nodes of the graph is calculated as edges of the graph. Correlation between the nodes could be calculated using 
various methods. Our data is computed using Pearson correlation coefficient. Normalization of edges has been implemented using Fisher Z-transformation.

Thresholding of the networks is a challenging step due to its important role on network extraction. The defined threshold should maintain a sufficient number of edges in the network and does not make an over-dense network simultaneously. In this research, instead of choosing one single threshold for all networks, we use network density, the proportion of the total number of existing edges on a network to the total number of possible edges, to obtain a variety of thresholds in the networks. We used network densities of $0.15-0.32$ with a step of 0.02 for thresholding. The lower limit is a sparsity level where the network becomes fragmented and network properties begin to break down. The upper threshold of $32 \%$ was chosen because the weakest edge it includes corresponds minimum correlation needed to be statistically significant.

\subsection{Feature extraction}

As mentioned before, we used a set of graphlet-based network features for classifying the data. Graphlets are connected non-isomorphic subgraphs which are defined as parts of a graph. In [13], 29 basic graphlets are introduced by Przulj et al. and multiple network metrics are extracted using this concept. We used a graphlet frequency vector as an indicator of healthy and Autism affected subjects. Graphlet frequency vector includes the counts of each 29 basic graphlets in the brain complex network.

Graphlet frequency vector is calculated for each of network density in the selected range. In order to accumulate the summery of the metric for each network, integral of graphlet frequencies is computed. As a result, a 29-graphlet frequency vector for each network is attained. For further investigations, the average of graphlet frequencies for each group of data is evaluated (Fig. 1). A notable point in Fig. 1 is the difference of graphlet frequency between ASD and TD subjects. This differentiation is more significant in some special graphlet frequencies, for instance, graphlet number 9 and 13. According to this point, 18 graphlets of 29 basic graphlets, which had remarkable alterations, are selected as a feature vector. These 18 graphlets are presented on Fig. 2 .

\subsection{Classification}

Selected graphlet frequencies are calculated for all subjects, normalized and presented to a classifier. After training and test phases, the classifier is able to predict the label of presented data from two groups of ASD and TD. Multiple classifiers, including K-nearest neighbor, decision trees, support vector machines (SVM) and ensemble

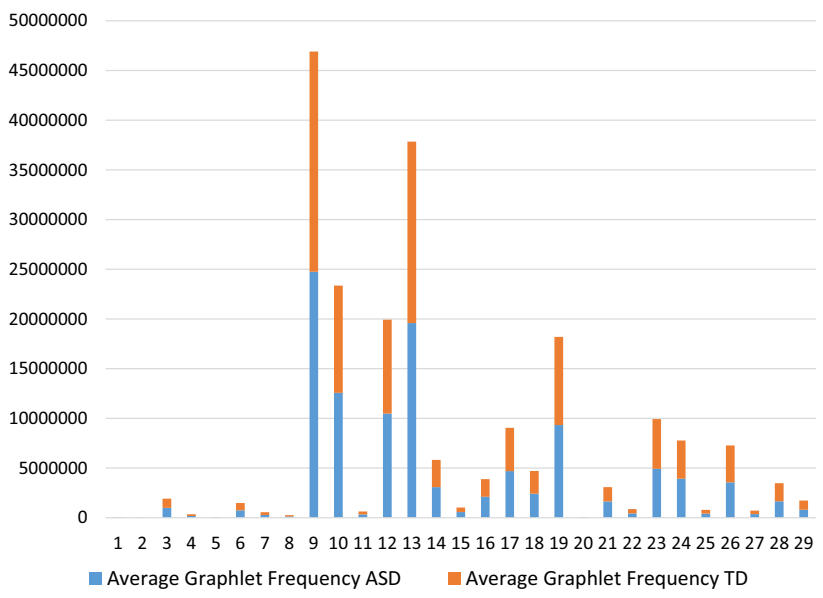

Fig. 1 Average graphlet frequency vector for autism spectrum disorder (ASD) and typically developing (TD) groups

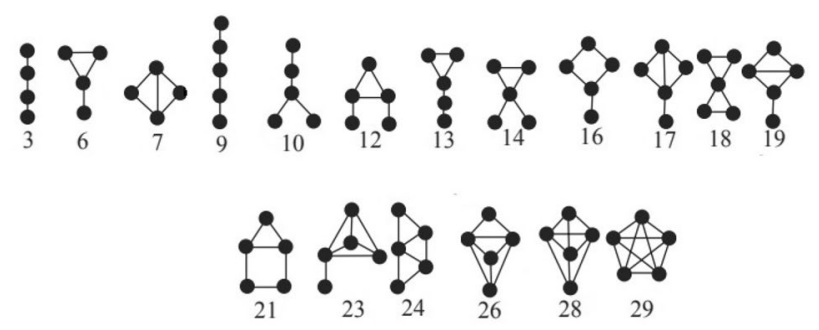

Fig. 218 selected graphlets as feature vector

methods, are used for classification of this dataset. In the classification process, fivefold cross-validation is implemented, and due to the small size of the dataset, the classification process is repeated 100 times with each of the classifiers to get the best accuracy.

\section{Results and discussion}

Average accuracy for 100 times of each classifier is presented on Table 2. All the classifiers have been trained and tested using fivefold cross-validation. As mentioned in Table 2, highest accuracy of $69.81 \%$, is reached using Ensemble Bagged Trees classifier. On this classifier 30 Decision trees are ensembled using bagging technique. According to the results, we propose Ensemble Bagged Decision Trees as most accurate classifier for this problem.

In this research, a method is presented for screening Autism using brain network graphlet frequency vector. According to the results, our approach outperforms both baseline methods presented in Sect. 2. Riemannian and Grassman Manifold approaches reported $60.76 \%$ and $63.29 \%$ respectively. Our method improved the accuracy by $6.5 \%$ and reached $69.81 \%$ average accuracy in 100 
Table 2 Average classification accuracy

\begin{tabular}{lll}
\hline Classifier type & Classifier name & $\begin{array}{l}\text { Average } \\
\text { accuracy } \\
(\%)\end{array}$ \\
\hline Decision tree & Bagged trees & $\mathbf{6 9 . 8 1}$ \\
& RUS boosted Trees & 66.37 \\
K nearest neighbor (KNN) & Fine KNN & 66.48 \\
& Weighted KNN & 63.24 \\
Support vector machine (SVM) & Subspace KNN & 63.06 \\
& Gabic SVM & 64.21 \\
\hline
\end{tabular}

Bootstrap aggregating, also called bagging, is a machine learning ensemble meta-algorithm designed to improve the stability and accuracy of machine learningalgorithms used in statistical classification and regression. It also reduces variance and helps to avoid overfitting. Although it is usually applied to decision treemethods, it can be used with any type of method. Bagging is a special case of the model averaging approach

times execution. Beside the main results, observation of a similar pattern for graphlet frequency fluctuations in both groups was a remarkable outcome of in this study.

\section{Compliance with ethical standards}

Conflict of interest The authors declare that they have no conflict of interest.

\section{References}

1. Brown JA, Rudie JD, Bandrowski A, Van Horn JD, Bookheimer SY (2012) The ucla multimodal connectivity database: a webbased platform for brain connectivity matrix sharing and analysis. Front Neuroinform 6:28

2. Dodero L, Minh HQ, San Biagio M, Murino V, Sona D (2015) Kernel-based classification for brain connectivity graphs on the riemannian manifold of positive definite matrices. In: 2015 IEEE 12th international symposium on biomedical imaging (ISBI). IEEE, pp 42-45
3. Dodero L, Sambataro F, Murino V, Sona D (2015) Kernel-based analysis of functional brain connectivity on grassmann manifold. In: International conference on medical image computing and computer-assisted intervention. Springer, pp 604-611

4. Fornito A, Zalesky A, Bullmore E (2016) Fundamentals of brain network analysis. Academic Press, Cambridge

5. Fox MD, Snyder AZ, Vincent JL, Corbetta M, Van Essen DC, Raichle ME (2005) The human brain is intrinsically organized into dynamic, anticorrelated functional networks. Proc Natl Acad Sci 102(27):9673-9678

6. Johnson CP, Myers SM (2007) Identification and evaluation of children with autism spectrum disorders. Pediatrics 120(5):1183-1215

7. Kanner $L$ (1949) Problems of nosology and psychodynamics of early infantile autism. Am J Orthopsychiatry 19(3):416

8. Kennedy DP, Courchesne E (2008) The intrinsic functional organization of the brain is altered in autism. Neuroimage 39(4):1877-1885

9. Lee MH, Smyser CD, Shimony JS (2013) Resting-state fMRI: a review of methods and clinical applications. Am J Neuroradiol 34(10):1866-1872

10. Newman M (2018) Networks. Oxford University Press, Oxford

11. Park HJ, Friston K (2013) Structural and functional brain networks: from connections to cognition. Science 342(6158):1238411

12. Power JD, Cohen AL, Nelson SM, Wig GS, Barnes KA, Church JA, Vogel AC, Laumann TO, Miezin FM, Schlaggar BL et al (2011) Functional network organization of the human brain. Neuron 72(4):665-678

13. Pržulj N, Corneil DG, Jurisica I (2004) Modeling interactome: scale-free or geometric? Bioinformatics 20(18):3508-3515

14. Rudie JD, Brown J, Beck-Pancer D, Hernandez L, Dennis E, Thompson P, Bookheimer S, Dapretto M (2013) Altered functional and structural brain network organization in autism. Neurolmage Clin 2:79-94

15. Sadeghi M, Khosrowabadi R, Bakouie F, Mahdavi H, Eslahchi C, Pouretemad H (2017) Screening of autism based on task-free fmri using graph theoretical approach. Psychiatry Res Neuroimaging 263:48-56

16. Shattuck PT, Seltzer MM, Greenberg JS, Orsmond GI, Bolt D, Kring S, Lounds J, Lord C (2007) Change in autism symptoms and maladaptive behaviors in adolescents and adults with an autism spectrum disorder. J Autism Dev Disord 37(9):1735-1747

Publisher's Note Springer Nature remains neutral with regard to jurisdictional claims in published maps and institutional affiliations. 